\title{
Antar, le gueux qui porte un espoir
}

\section{Résumé de recherche préparé par}

Doaa Hosny Abdelkhalek

Maître assistante, faculté des lettres. Université d'Assuit

Sous la direction de

\section{Gusine Gawdat}

Professeur de critique littéraire et de littérature française et francophone.

Université du Caire

\author{
Khalaf Abdelaziz \\ Professeur adjoint de littérature comparée
}

\section{Antar le gueux qui porte un espoir}

Antara (ou Antar dans les traditions populaires) était le fils de Shaddad l'un des chefs de la tribu de Béni Abs. Quant à sa mère, elle était «une esclave noire abyssine nommé Zabiba ${ }^{1}$ ». Le statut de sa mère le mettait au rang des esclaves. Il était donc le résultat de l'alliance de deux sangs différents, de deux couleurs différentes, et notamment de deux conditions sociales bien différentes.

Esclave, noir et considéré comme bâtard, mais distingué par sa bravoure et sa force physique grâce auxquelles il obtient son émancipation, la reconnaissance de son père et la main de celle qu'il aime. Telle est l'histoire d'un gueux bédouin qui, par sa persévérance, son don poétique exceptionnel et ses prouesses guerrières, devient le héros des arabes par excellence.

Antara : sa double exclusion et sa double origine

Antara a souffert d'abord d'une double exclusion. «Il est exclu parce qu'il appartient à une caste inférieure celle de l'esclave. Il est aussi marginalisé parce qu'il est noir» ${ }^{2}$.Nous avons mentionné plus haut que dans ce temps là, les arabes ne reconnaissaient pas leur fils nés d'une mère esclave. Le destin d'Antar est donc de rester esclave pour toujours sans père légitime, en raison de son rang social inférieur et de la couleur foncée de sa peau.

Antar ne pouvait obtenir le seul prix qu'il ambitionnait, le titre de fils reconnu et légitime de son père Sheddad. Vil bâtard, lui dit Sheddad, oses-tu bien prétendre au rang de mes autres fils; toi, fils d'une esclave, toi qui portes la honte de ta naissance écrite sur ta peau ${ }^{3}$

Le père de Antar agissait donc comme la plupart des seigneurs de son temps, il a refusé de donner son nom à son fils tout en le mettant au rang de serviteurs qui doivent garder les troupeaux. Certes l'origine servile de Antaral'a mis au rang de 1905 p.5.

${ }^{1}$ Hartwig Derenbourg, Opuscule d'un arabisant, Paris, Charles Carrington, Libraire,

${ }^{2}$ Driss Cherkaoui,Le roman de Antar: Une perspective littéraire et historique, France, présence Africaine, 2011,p.

${ }^{3}$ Alphonse de Lamartine, Antar, op.cit, p.85 
serviteur, il passa la plus grande partie de sa jeunesse à garder les troupeaux de son père.

L'enfant participant de sa double origine, fils d'un chef libre et d'une esclave préférée, fut traité par son père tantôt en serviteur, tantôt en fils. Il gardait les troupeaux dans la solitude. ${ }^{1}$

En effet, la couleur de la peau de Antara et son origine servile constituent un thème majeur auquel se rattachent de nombreux événements de récit. Hérité de sa mère la noirceur de la peau, il était rangé parmi les corbeaux des arabes. «Plusieurs poètes de l'ère préislamique(Djâhiliya) avaient hérité de leur mère un teint foncé qui leur valut le surnom d'Aghribat al Arab les corbeaux des arabes, les plus célèbres d'entre eux sont Antar» ${ }^{2}$

En raison de cette pigmentation négroïde de sa peau, il a été non seulement mis au nombre de corbeaux, et n'était pas reconnu de son père, mais il était condamné à protéger toute sa vie le campement des femmes et garder les troupeaux en l'absence des hommes de la tribu, sans pouvoir ambitionner de guerroyer à côté des princes et des hommes libres et de porter les armes de combattants arabes.

Pendant l'absence de tous les guerriers de la tribu d'Abs, partant pour une expédition lointaine, on confia les femmes, les enfants, les vieillards, les troupeaux, les trésors, les tentes à la garde du seul Antar3.

En effet, Les aspects de l'exclusion se révèlent dans plusieurs cotés de la vie de Antara, nous ne pouvons pas parler de cette exclusion sans évoquer l'amour malheureux de Antar pour sa cousine Abla. Cet amour proverbial était d'abord difficile à concrétiser c'était également pour la même raison, celle de la naissance honteuse de Antara

\section{Antar et Abla: La différence de classe ou la tare sociale}

Antar était amoureux de sa cousine Abla. Il est le fils d'une esclave noire tandis qu'Abla est une jeune fille libre d'origine noble. Même que cette dernière fait partie de sa famille proche, l'orgueil arabe refuse obstinément ce mariage en raison de cette tare sociale.

\section{L'orgueil arabe se révolte à l'idée de consentir à l'union d'une fille libre et d'un esclave noir ${ }^{4}$}

Aux yeux de Malik et aux yeux des arabes, cet amour était une sorte de sacrilège et c'était un déshonneur qu'un notable donne sa fille à un homme de piètre condition même s'il était le poète le plus éminent dans sa tribu

Antar ne se dissimulait pas que son amour pour Ablaétait, aux yeux des arabes, une sorte de sacrilège, et que Malek, père de Abla, n'accorderait jamais sa fille, à moins de miracles, à un enfant marqué de la couleur de la servitude. ${ }^{5}$

\footnotetext{
${ }^{1}$ Alphonse de Lamartine, Antar, op.cit, p.71

${ }^{2}$ M.El Fasi, Histoire générale de l'Afrique, III: L'Afrique du VIIe au

${ }^{3}$ Alphonse de Lamartine, Antar, op.cit, p.80

${ }^{4}$ Alphonse de Lamartine, Antar, op.cit, p.86

${ }^{5}$ Ibid. p. 77
} XIesiècle,Paris,UNESCO,1997, p.485 
En effet, le rang social de Antara non seulement l'a empêché de gagner le cœur de Abla, mais il a aussi provoqué la haine de ses oncles qui dressent milles embûches pour l'assassiner. Afin de se soustraire donc à une telle alliance déshonorant, ils l'ont envoyé maintes fois dans un lieu périlleux où ils espéraient qu'il trouverait la mort.

Les oncles d'Abla humiliés et irrités de ce qu'un vil esclave noir osait lever les yeux sur elles, tendent mille pièges à l'adolescent pour le faire succomber, tantôt contre les guerriers, tantôt contre les bêtes féroces de déserts. Sa force et son courage déjouent toujours leurs embûches. ${ }^{1}$

Certes, la différence de classe entre Antar et Abla mettait obstacle à l'union de deux amants, mais si l'espritde la société dans laquelle vivait Antar était le premier responsable de cette obstacle, on trouve que la résistance et la persévérance de Antara viennent vaincre toute obstacle. C'est pour Abla qu'il décide de forcer son destin, c'est pour Abla qu'il ambitionnait de quitter sa condition sociale défavorisée pour accéder au statut d'homme libre. En outre, si nous parcourons le roman de Antar nous trouvons que la trame essentielle du récit est celle du challenge de Antara pour gagner la main de Abla«tout le roman de Antara est bâti sur ce thème : l'ancien esclave noir qui s'est imposé par sa bravoure et ses haut faits, convoite désespérément la main de sa cousine blanche $»^{2}$. En d'autres termes l'amour d'Antar pour sa bien aiméeAbla«est le mobile principale de ses actions, de ses faits d'armes, c'est pour elle qu'il combat, soupire et chante»3. Ainsi, notre héros exprime:

Abla! Abla! Tu es le seul rêve de mon cœur et ne cherche la renommée que pour ne pas été méprisé un jour par toi! Je suis noir, oui mais j'en suis sur j'écraserai l'envie, j'anéantirai tout ce qui osera me résister. Je combats pour Abla, je suis son esclave ${ }^{4}$

Après avoir révélé le côté obscur de la vie de Antara, il convient maintenant d'aborder le côté lumineux et favorable. Si Antara est frappé d'abord par le phénomène d'exclusion et de la marginalisation, s'il vivait dan un milieu social où il a été rejeté, isolé, repoussé. Petit à petit, il échappe complètement à sa condition humiliante et devient un membre vénéré dans cette société. En parcourant donc le Roman d'Antara, nous y décelons deux conditions bien différentes «le Roman d'Antar présente le développement d'une grande moralité: on y voit un homme privé des avantages de la figure et de la naissance, qui parvient cependant par la force d'âme, par la puissance d'esprit et par son indomptable courage, à être jugé digne du premier rang parmi les hommes $\gg^{5}$

Ecartelé entre la réalité qu'on lui impose et celle qu'il souhaite vivre, Antara a contesté de se soumettre aux dures conditions que la société lui inflige. Là nous

${ }^{1}$ Ibid. p.79

${ }^{2}$ JosepheChelhod, Le droit dans la société Bédouine, Paris, Librairie Marcel Rivière, 1971, p.22.

${ }^{3}$ J.J Ampère,Mélanges d'histoires littéraires et de littérature, Paris, Michel Lévy frères, Libraires, 1867, p.171.

${ }^{4}$ Alphonse de Lamartine, Antar, op.cit, p.82.

${ }^{5}$ Etienne Jean Délécluze,Roland ou la chevalerie, Paris, Jules Labittes, Libraire, 1845, p. 311 
pouvons dire qu'il ya une nouvelle étape qui apparait bel et bien dans le parcours du héros, cette étape très importante est celle de la vengeance ou la revanche sociale. En effet, la vengeance est une manière de se faire justice ou de la rétablir. Ce n'est pas forcément la vengeance qui consiste à punir les autres, mais c'est plutôt la revanche du héros sur le destin, celle-ci devient alors une force destinée à construire quelque chose. Antara, Guillaume Tell, Robin de Bois, Ali El Zaybek, tous ces héros et bien d'autres héros populaire sont un passé malheureux qui a donné lieu à des représailles, la propension à la vengeance est donc tributaire d'un passé miséreux où l'on veut passer de l'errance à l'affirmation de soi; de la honte à la fierté, de la tristesse à la joie.

\section{Du rejet total à l'ascension sociale: la revanche sociale}

Dans cette phase, Antara a donné l'exemple le plus parfait, car tout simplement c'est l'histoire d'un gueux esclave qui devient plus tard «le parangon du caractère masculin arabe» ${ }^{1}$. Dans son histoire extraordinaire toutes les classes défavorisées, qui n'ont connu de la société que toute sorte de l'injustice, voit certainement un lendemain lumineux. Autrement dit « la revanche extraordinaire du héros sur le destin a certainement dû réconforter les générations d'auditeurs--pauvres gens, étrangers, esclaves, mais aussi amoureux éconduits ou même pourquoi pas notables en disgrâce-- dans le monde arabe, leur donner la force de mieux vivre leur sort malheureux en espérant qu'il ne soit que passager ${ }^{2} \gg$.

Certes, ascension sociale et revanche sociale sont deux termes qui sont intimement liés car si Antara, cet homme, qui a tant souffert de sa couleur de peau, du mépris et du dénigrement de la société toute entière, peut parvenir un jour au sommet de l'échelle, le peuple voit certainement dans cette ascension une victoire sur l'autre et une affirmation de soi dans un monde hostile et injuste. Par conséquent, ce peuple trouve enfin que cet homme, qui finit par briser tout seul ces obstacles, n'était pas un simple homme mais, c'est son héros populaire.

En effet, Antara ne reste pas passif face aux piètres conditions, mais il se sert de toutes ses qualités brillantes que la nature l'avait doué pour changer sa vie. Grâce à sa performance chevaleresque, son talent poétique et ses actes valeureux, il est devenu non seulement un homme libre mais aussi le cavalier des cavaliers des arabes.

Des conditions très dures à réaliser mais Antar relève toujours le défi et il réussit chaque fois. Nous avons mentionné plus haut qu'Antar n'avait pas le droit de combattre. Tandis que les hommes libres guerroient, il est réduit à protéger le campement des femmes. Mais petit à petit, il a montré à son père Shaddad qu'il mérite toute confiance. Ainsi il a participé à de nombreuses batailles dont il revient chaque fois vainqueur. Désormais il a cessé d'être compté parmi les serviteurs de son père

1 XavierLuffin, «La tradition épique arabe, un genre à (re-)découvriren Occident », in Bon à tirer, 2011, $\mathrm{n}^{\circ} 152$

${ }^{2}$ Ibid. 
Schédad délia son fils, pleura de joie et l'orgueil au récit de ses exploits, et le conduisit au roi Zobéir qui l'admit au rang des guerriers, de ce jour Antara cessa de faire partie parmi les esclaves de Schédad ${ }^{1}$

Antar a conquit donc sa liberté, sa gloire ainsi que la main de celle qu'il aime ayant apporté la dot exigée de son oncle:

Malek, son oncle, vaincu par ce retour triomphal, lui accorda sa fille. L'esclave noir, enrichi de dons de Moundhir et les Chosroés, devine le plus puissant et le plus opulent des arabes de la tribu d'Abs. ${ }^{2}$

Ainsi se termine l'histoire d'Antar d'une fin heureuse comme le veut l'imaginaire populaire arabe. C'est de cette grande victoire accomplie par ce héros intrépide sur ses complexes que le menu peuple peut trouver un modèle qui a reculé les limites de l'impossible il n'ya là rien de surprenant car Antara a vraiment triomphé de toutes les oppositions et toutes les résistances «il parvient, à force de prouesses à triompher de toutes les résistances, se fait reconnaître par son père, et, admis au rang des nobles, épouse celle qu'il aime et devient le premier de sa tribu, qui est la première parmi les nomades de l'Arabie» ${ }^{3}$

En effet, ce récit populaire non seulement a redonné l'espoir et l'optimisme au menu peuple ou les gens qui ne trouvent pas leur place dans la vie, mais elle a restitué aussi la gloire aux arabes (notables en disgrace) «la sîra de Antara aurait vu le jour à une époque où les arabes sentirent que leur existence, leur ethnie, leurs gloires et leurs valeurs étaient en danger de disparition et ce, à la fin de la dynastie abbasside. Afin de faire face à ce danger, ils se réfugièrent dans leur passé pour y retrouver leurs valeurs, leurs gloires et leur suprématie. L'art populaire fut, en l'occurrence, pour les arabes un moyen efficace de se représenter leur histoire glorieuse et leurs espoirs à travers la sîra de Antar» ${ }^{4}$

${ }^{1}$ Alphonse de Lamartine, Antar, op.cit, p.85

${ }^{2}$ Ibid. p. 147

${ }^{3}$ L. Marcel Devic, Lesaventures de Antar fils de Cheddad, roman arabedes temps antéislamiques, ParisJ. Hetzel, 1864, p. xi

${ }^{4}$ BasmaNouha Chaouch, L'image d'Abla entre la sîra et la poésie d'Antara, universitéde Tunis, $2009, \mathrm{n}^{\circ} 6$ 